\title{
MARCINKIEWICZ INTEGRALS ALONG SUBVARIETIES ON PRODUCT DOMAINS
}

\author{
AHMAD AL-SALMAN \\ Received 25 January 2004
}

\begin{abstract}
We study the $L^{p}$ mapping properties of a class of Marcinkiewicz integral operators on product domains with rough kernels supported by subvarieties.
\end{abstract}

2000 Mathematics Subject Classification: 42B20, 42B15, 42B25.

1. Introduction. Let $\mathbb{R}^{d}(d=n$ or $d=m), d \geq 2$ be the $d$-dimensional Euclidean space and $\mathbf{S}^{d-1}$ be the unit sphere in $\mathbb{R}^{d}$ equipped with the induced Lebesgue measure $d \sigma$. Suppose that $\Omega$ is a homogeneous function of degree zero on $\mathbb{R}^{n}$ which is integrable on $\mathbf{S}^{n-1}$ and $\int_{\mathbf{S}^{n-1}} \Omega(y) d \sigma(y)=0$. Then the Marcinkiewicz integral operator $\mu_{\Omega}$ which was introduced by Stein in [18] is defined by

$$
\mu_{\Omega}(f)(x)=\left(\left.\left.\int_{-\infty}^{\infty}\left|\int_{|y| \leq 2^{t}} f(x-y) \Omega(y)\right| y\right|^{1-n} d y\right|^{2} 2^{-2 t} d t\right)^{1 / 2} .
$$

Stein proved that if $\Omega \in \operatorname{Lip}_{\alpha}\left(\mathbf{S}^{n-1}\right),(0<\alpha \leq 1)$, then $\mu_{\Omega}$ is bounded on $L^{p}$ for all $1<p \leq$ 2 [18]. Since then, the study of the $L^{p}$ boundedness of $\mu_{\Omega}$ under various conditions on the function $\Omega$ has attracted the attention of many authors ([1, 4, 5, 7, 10, 13], among others). In particular, Chen et al. in [8] studied the $L^{p}$ boundedness of $\mu_{\Omega}$ under the following condition on the function $\Omega$ which was introduced by Grafakos and Stefanov in their study of singular integral operators [17]:

$$
\sup _{\xi \in \mathbf{S}^{n-1}} \int_{\mathbf{S}^{n-1}}\left|\Omega\left(y^{\prime}\right)\right|\left(\log \frac{1}{\left|\xi \cdot y^{\prime}\right|}\right)^{1+\alpha} d \sigma\left(y^{\prime}\right)<\infty
$$

for some $\alpha>0$. Chen et al. [8] showed that if $\Omega$ satisfies (1.2) for some $\alpha>0$, then $\mu_{\Omega}$ is bounded on $L^{p}$ for $p \in((2+2 \alpha) /(1+2 \alpha), 2+2 \alpha)$. It should be pointed out here that Grafakos and Stefanov showed that for any $\alpha>0$, the following relations hold:

$$
F\left(\alpha, \mathbf{S}^{n-1}\right) \nsubseteq L\left(\log ^{+} L\right)\left(\mathbf{S}^{n-1}\right), \quad L\left(\log ^{+} L\right)\left(\mathbf{S}^{n-1}\right) \nsubseteq F\left(\alpha, \mathbf{S}^{n-1}\right),
$$

where $F\left(\alpha, \mathbf{S}^{n-1}\right)$ is the space of all integrable functions $\Omega$ on $\mathbf{S}^{n-1}$ which satisfy $\int_{\mathbf{S}^{n-1}} \Omega(y) d \sigma(y)=0$ and (1.2). For conditions similar to (1.2), we refer the readers to consult $[1,6]$. 
Recently, a number of authors started to study the analog of the operator $\mu_{\Omega}$ on product domains. More precisely, let $\Omega \in L^{1}\left(\mathbf{S}^{n-1} \times \mathbf{S}^{m-1}\right)$ be such that

$$
\begin{gathered}
\Omega(t x, s y)=\Omega(x, y) \text { for any } t, s>0, \\
\int_{S^{n-1}} \Omega(u, \cdot) d \sigma(u)=\int_{S^{m-1}} \Omega(\cdot, v) d \sigma(v)=0 .
\end{gathered}
$$

Then, the Marcinkiewicz integral operator on product domains $M_{\Omega, c}$ is given by

$$
\mu_{\Omega, c} f(x, y)=\left(\int_{-\infty}^{\infty} \int_{-\infty}^{\infty}\left|F_{t, s}(f)(x, y)\right|^{2} 2^{-2(t+s)} d t d s\right)^{1 / 2}
$$

where

$$
F_{t, s}(f)(x, y)=\int_{|u| \leq 2^{t}} \int_{|v| \leq 2^{s}} f(x-u, y-v) \frac{\Omega(u, v) d u d v}{|u|^{n-1}|v|^{m-1}} .
$$

It has been known for quite some time that the operator $M_{\Omega, c}$ is bounded on $L^{p}$ for all $1<p<\infty$ under the condition that $\Omega \in L\left(\log ^{+} L\right)^{2}\left(\mathbf{S}^{n-1} \times \mathbf{S}^{m-1}\right)[9,12]$. Recently, the $L^{p}$ boundedness of $\mu_{\Omega, c}$ was established under the weaker condition $\Omega \in L\left(\log ^{+} L\right)\left(\mathbf{S}^{n-1} \times\right.$ $\mathbf{S}^{m-1}$ ); see Choi [11] for $p=2$ and Al-Qassem et al. [2] for all $1<p<\infty$.

Motivated by $[1,6]$, the main purpose of this note is to investigate the $L^{p}$ boundedness of Marcinkiewicz integral operators on product domains with kernels satisfying conditions similar to (5) in [6] (see also [1]) and supported by subvarieties determined by polynomial mappings. To be more specific, let $\mathscr{V}(d, l)$ be the set of real-valued polynomials in $\mathbb{R}^{d}$ which have degrees at most $l$. For $\mathscr{P}=\left(P_{1}, \ldots, P_{N}\right) \in(\mathscr{V}(d, l))^{N}$ and $\mathscr{2}=$ $\left(Q_{1}, \ldots, Q_{M}\right) \in(\mathscr{V}(d, r))^{M}$, consider the Marcinkiewicz integral operator

$$
M_{\Omega, \mathscr{P}, \mathscr{Q}} f(x, y)=\left(\int_{-\infty}^{\infty} \int_{-\infty}^{\infty}\left|F_{t, s}^{\mathscr{P}, \mathcal{L}}(f)(x, y)\right|^{2} 2^{-2(t+s)} d t d s\right)^{1 / 2},
$$

where

$$
F_{t, s}^{\mathscr{P}, \mathscr{Q}}(f)(x, y)=\int_{|u| \leq 2^{t}} \int_{|v| \leq 2^{s}} f(x-\mathscr{P}(u), y-2(v)) \frac{\Omega(u, v) d u d v}{|u|^{n-1}|v|^{m-1}} .
$$

It is clear that if $N=n, M=m, \mathscr{P}(u)=u$, and $\mathscr{2}(v)=v$, then $M_{\Omega, \mathscr{P}, 2}=M_{\Omega, c}$. In order to formulate our main results regarding the operators $(1.7)$, we let $\mathscr{H}(d, l)$ be the collection of all homogeneous polynomials of degree $l$ in $\mathscr{V}(d, l)$. We will associate with $\mathscr{V}(d, l)$ the norm $\|\cdot\|$ given by $\|P\|=\left(\sum_{|\beta| \leq d}\left|a_{\beta}\right|^{2}\right)^{1 / 2}$, where $P(y)=\sum_{|\beta| \leq d} a_{\beta} y^{\beta}$. Let

$$
\mathscr{H}^{I}(d, l)=\{P \in \mathscr{H}(d, l):\|P\|=1\} .
$$

Definition 1.1. For $n, m \geq 2, l, r \in \mathbb{N}$, and $\alpha>0$, let $F_{\alpha}\left(\mathbf{S}^{n-1}, \mathbf{S}^{m-1}, l, r\right)$ be the space of all integrable functions $\Omega$ on $\mathbf{S}^{n-1} \times \mathbf{S}^{m-1}$ that satisfy

$$
\sup _{P \in \mathscr{H}^{I}(n, l), Q \in \mathscr{H}^{I}(m, r)} \int_{\mathbf{S}^{n-1}} \int_{\mathbf{S}^{m-1}}\left|\Omega\left(u^{\prime}, v^{\prime}\right)\right|\left\{G_{P, Q}\left(u^{\prime}, v^{\prime}\right)\right\}^{1+\alpha} d \sigma\left(u^{\prime}\right) d \sigma\left(v^{\prime}\right)<\infty,
$$


where $\alpha>0$ and

$$
\begin{aligned}
G_{P, Q}\left(u^{\prime}, v^{\prime}\right)= & \log ^{+}\left(\left|P\left(u^{\prime}\right)\right|^{-1}\right)+\log ^{+}\left(\left|Q\left(v^{\prime}\right)\right|^{-1}\right) \\
& +\log ^{+}\left(\left|P\left(u^{\prime}\right)\right|^{-1}\right) \log ^{+}\left(\left|Q\left(v^{\prime}\right)\right|^{-1}\right) .
\end{aligned}
$$

It is clear that $L^{q}\left(\mathbf{S}^{n-1} \times \mathbf{S}^{m-1}\right) \varsubsetneqq F_{\alpha}\left(\mathbf{S}^{n-1}, \mathbf{S}^{m-1}, l, r\right)$ for all $q>1$. Moreover, by (1.3) it can be easily shown that

$$
\begin{gathered}
F_{\alpha}\left(\mathbf{S}^{n-1}, \mathbf{S}^{m-1}, l, r\right) \nsubseteq L\left(\log ^{+} L\right)\left(\mathbf{S}^{n-1} \times \mathbf{S}^{m-1}\right), \\
L\left(\log ^{+} L\right)\left(\mathbf{S}^{n-1} \times \mathbf{S}^{m-1}\right) \nsubseteq F_{\alpha}\left(\mathbf{S}^{n-1}, \mathbf{S}^{m-1}, l, r\right)
\end{gathered}
$$

for any $\alpha>0$. Therefore, by (1.12) and the results of Choi [11] and Al-Qassem et al. [2] when $\Omega \in L\left(\log ^{+} L\right)\left(\mathbf{S}^{n-1} \times \mathbf{S}^{m-1}\right)$, it is natural to investigate the $L^{p}$ boundedness of $M_{\Omega, \mathscr{P}, 2}$ under the conditions (1.10).

Our main result is the following.

TheOREM 1.2. Let $n, m \geq 2, N, M, \tilde{N}, \tilde{M} \in \mathbb{N}, \mathscr{P}=\left(P_{1}, \ldots, P_{N}\right) \in(\mathscr{V}(n, \tilde{N}))^{N}$, and $2=$ $\left(Q_{1}, \ldots, Q_{M}\right) \in(\mathscr{V}(m, \tilde{M}))^{M}$. If $\Omega$ satisfies (1.4) and

$$
\Omega \in \bigcap_{l=1}^{\tilde{N}} \bigcap_{r=1}^{\tilde{M}} F_{\alpha}\left(\mathbf{S}^{n-1}, \mathbf{S}^{m-1}, l, r\right)
$$

for some $\alpha>0$, then

$$
\left\|M_{\Omega, \mathscr{P}, 2} f\right\|_{L^{p}\left(\mathbb{R}^{N} \times \mathbb{R}^{M}\right)} \leq C_{p}\|f\|_{L^{p}\left(\mathbb{R}^{N} \times \mathbb{R}^{M}\right)}
$$

for all $p \in((2+2 \alpha) /(1+2 \alpha), 2+2 \alpha)$ and $f \in L^{p}\left(\mathbb{R}^{N} \times \mathbb{R}^{M}\right)$. The constant $C_{p}$ is independent of the coefficients of the polynomials $\left\{P_{j}, Q_{k}: 1 \leq j \leq N, 1 \leq k \leq M\right\}$.

In Section 4, we will show that

$$
\bigcap_{l=1}^{\infty} \bigcap_{r=1}^{\infty} F_{\alpha}\left(\mathbf{S}^{1}, \mathbf{S}^{1}, l, r\right)=F_{\alpha}\left(\mathbf{S}^{1}, \mathbf{S}^{1}, 1,1\right) .
$$

Therefore, we obtain the following.

COROLlary 1.3. Let $N, M, \tilde{N}, \tilde{M} \in \mathbb{N}, \mathscr{P}=\left(P_{1}, \ldots, P_{N}\right) \in(\mathscr{V}(2, \tilde{N}))^{N}$, and $\mathscr{2}=\left(Q_{1}, \ldots, Q_{M}\right)$ $\in(\mathscr{V}(2, \tilde{M}))^{M}$. If $\Omega$ satisfies (1.4) and $\Omega \in F_{\alpha}\left(\mathbf{S}^{1}, \mathbf{S}^{1}, 1,1\right)$ for some $\alpha>0$, then $\left\|M_{\Omega, \mathscr{P}, \mathcal{Q}} f\right\|_{L^{p}\left(\mathbb{R}^{N} \times \mathbb{R}^{M}\right)} \leq C_{p}\|f\|_{L^{p}\left(\mathbb{R}^{N} \times \mathbb{R}^{M}\right)}$ for all $p \in((2+2 \alpha) /(1+2 \alpha), 2+2 \alpha)$ and $f \in$ $L^{p}\left(\mathbb{R}^{N} \times \mathbb{R}^{M}\right)$. The constant $C_{p}$ is independent of the coefficients of the polynomials $\left\{P_{j}, Q_{k}: 1 \leq j \leq N, 1 \leq k \leq M\right\}$.

2. General tools. For a nonnegative $C^{\infty}$ radial function $\Phi$ on $\mathbb{R}$ and a linear transformation $L: \mathbb{R}^{d} \rightarrow \mathbb{R}^{l}$, we let $\check{\Phi}_{w}^{L}$ be such that $\left(\check{\Phi}_{w}^{L}\right)^{\wedge}(\xi)=\Phi\left(|w L(\xi)|^{2}\right)$. Let $N, M \geq 2$ and let $\sigma=\left\{\sigma_{t, s}: t, s \in \mathbb{R}\right\}$ be a family of measure defined on $\mathbb{R}^{N} \times \mathbb{R}^{M}$. For linear transformations $L: \mathbb{R}^{N} \rightarrow \mathbb{R}^{\tilde{N}}, G: \mathbb{R}^{M} \rightarrow \mathbb{R}^{\widetilde{M}}$, nonnegative $C^{\infty}$ radial function $\Phi$ on $\mathbb{R}$ with 
$\operatorname{supp}(\Phi) \subset\left[2^{-1}, 2\right]$ and $0 \leq \Phi(t) \leq 1$, and positive real numbers $a$ and $b$, define the family of operators $\left\{Z_{\theta, \gamma}^{(a, b)}: \theta, \gamma \in \mathbb{R}\right\}$ by

$$
Z_{\sigma, \theta, \gamma, \Phi, L, G}^{(a, b)} f(x, y)=\left(\int_{-\infty}^{\infty} \int_{-\infty}^{\infty}\left|\left(\check{\Phi}_{2^{a(t+\theta)}}^{L} \otimes \breve{\Phi}_{2^{b(s+\gamma)}}^{G}\right) * \sigma_{t, s} * f(x, y)\right|^{2} d t d s\right)^{1 / 2} .
$$

Also, define the operators $\mu_{\sigma, \Phi, L, G}^{(a, b, \infty,++)}, \mu_{\sigma, \Phi, L, G}^{(a, b, \infty,+-)}, \mu_{\sigma, \Phi, L, G}^{(a, b, \infty,--)}, \mu_{\sigma, \Phi, L, G}^{(a, b, \infty,-+)}, \mu_{\sigma, \Phi, L, G}^{(a, b, 0 \infty,+)}$, $\mu_{\sigma, \Phi, L, G}^{(a, b, 0 \infty,-)}, \mu_{\sigma, \Phi, L, G}^{(a, b, \infty 0,-)}, \mu_{\sigma, \Phi, L, G}^{(a, b, \infty 0,+)}$, and $\mu_{\sigma, \Phi, L, G}^{(a, b, 00)}$ by

$$
\begin{aligned}
\mu_{\sigma, \Phi, L, G}^{(a, b, \infty,++)} f(x, y) & =\int_{2}^{\infty} \int_{2}^{\infty} Z_{\sigma, \theta, \gamma, \Phi, L, G}^{(a, b)} f(x, y) d \theta d \gamma, \\
\mu_{\sigma, \Phi, L, G}^{(a, b, \infty,+-)} f(x, y) & =\int_{2}^{\infty} \int_{-\infty}^{-2} Z_{\sigma, \theta, \gamma, \Phi, L, G}^{(a, b)} f(x, y) d \theta d \gamma, \\
\mu_{\sigma, \Phi, L, G}^{(a, b, \infty,--)} f(x, y) & =\int_{-\infty}^{-2} \int_{-\infty}^{-2} Z_{\sigma, \theta, \gamma, \Phi, L, G}^{(a, b)} f(x, y) d \theta d \gamma, \\
\mu_{\sigma, \Phi, L, G}^{(a, b, \infty,-+)} f(x, y) & =\int_{-\infty}^{-2} \int_{2}^{\infty} Z_{\sigma, \theta, \gamma, \Phi, L, G}^{(a, b)} f(x, y) d \theta d \gamma, \\
\mu_{\sigma, \Phi, L, G}^{(a, b, 0 \infty,+)} f(x, y) & =\int_{-2}^{2} \int_{2}^{\infty} Z_{\sigma, \theta, \gamma, \Phi, L, G}^{(a, b)} f(x, y) d \theta d \gamma, \\
\mu_{\sigma, \Phi, L, G}^{(a, b, 0 \infty,-)} f(x, y) & =\int_{-2}^{2} \int_{-\infty}^{-2} Z_{\sigma, \theta, \gamma, \Phi, L, G}^{(a, b)} f(x, y) d \theta d \gamma, \\
\mu_{\sigma, \Phi, L, G}^{(a, b, \infty 0,-)} f(x, y) & =\int_{-\infty}^{-2} \int_{-2}^{2} Z_{\sigma, \theta, \gamma, \Phi, L, G}^{(a, b)} f(x, y) d \theta d \gamma, \\
\mu_{\sigma, \Phi, L, G}^{(a, b, \infty 0,+)} f(x, y) & =\int_{2}^{\infty} \int_{-2}^{2} Z_{\sigma, \theta, \gamma, \Phi, L, G}^{(a, b)} f(x, y) d \theta d \gamma, \\
\mu_{\sigma, \Phi, L, G}^{(a, b, 00)} f(x, y) & =\int_{-2}^{2} \int_{-2}^{2} Z_{\sigma, \theta, \gamma, \Phi, L, G}^{(a, b)} f(x, y) d \theta d \gamma .
\end{aligned}
$$

We also, let $M_{\sigma}$ denote the maximal function corresponding to $\sigma$, that is,

$$
M_{\sigma} f(x, y)=\sup _{t, s \in \mathbb{R}}|| \sigma_{t, s}|* f(x, y)| .
$$

LEMMA 2.1. Let $N, M, \tilde{N}, \widetilde{M} \geq 1, a, b \geq 1$, and $\alpha>0$. Let $\sigma=\left\{\sigma_{t, s}: t, s \in \mathbb{R}\right\}$ be a family of measure defined on $\mathbb{R}^{N} \times \mathbb{R}^{M}$ and let $\Phi$ be as above. Suppose that there exist linear transformations $L: \mathbb{R}^{N} \rightarrow \mathbb{R}^{\tilde{N}}$ and $G: \mathbb{R}^{M} \rightarrow \mathbb{R}^{\widetilde{M}}$ such that

(a) $\left\|\sigma_{t, s}\right\| \leq C$,

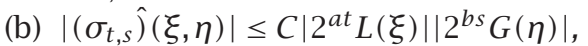

(c) $\left|\left(\sigma_{t, s}\right)(\xi, \eta)\right| \leq C\left|2^{a t} L(\xi)\right|\left(\log ^{+}\left|2^{b s} G(\eta)\right|\right)^{-1-\alpha}$,

(d) $\left|\left(\sigma_{t, s}\right)(\xi, \eta)\right| \leq C\left(\log ^{+}\left|2^{a t} L(\xi)\right|\right)^{-1-\alpha}\left(\log ^{+}\left|2^{b s} G(\eta)\right|\right)^{-1-\alpha}$,

(e) $\left|\left(\sigma_{t, s}\right)(\xi, \eta)\right| \leq C\left(\log ^{+}\left|2^{a t} L(\xi)\right|\right)^{-1-\alpha}\left|2^{b s} G(\eta)\right|$,

(f) $\left\|M_{\sigma}(f)\right\|_{L^{p}\left(\mathbb{R}^{N} \times \mathbb{R}^{M}\right)} \leq C\|f\|_{L^{p}\left(\mathbb{R}^{N} \times \mathbb{R}^{M}\right)}$ for all $1<p<\infty$.

Then the operators (2.2)-(2.10) are bounded on $L^{p}\left(\mathbb{R}^{N} \times \mathbb{R}^{M}\right)$ for all $p \in((2+2 \alpha) /(1+$ $2 \alpha), 2+2 \alpha)$. Moreover the $L^{p}$ bounds are independent of the linear transformations $L$ and $G$.

Proof. By the argument in $[3,15]$, we may assume that $\tilde{N} \leq N, \widetilde{M} \leq M, L(\xi)=$ $\left(\xi_{1}, \ldots, \xi_{\tilde{N}}\right)$, and $G(\eta)=\left(\eta_{1}, \ldots, \eta_{\widetilde{M}}\right)$ where $\xi=\left(\xi_{1}, \ldots, \xi_{N}\right)$ and $\eta=\left(\eta_{1}, \ldots, \eta_{M}\right)$. Let 
$\mathbf{J}_{\theta, \gamma, \Phi, L, G}^{(a, b)}$ be the operator given by

$$
\mathbf{J}_{\theta, \gamma, \Phi, L, G}^{(a, b)}(f)(x, y)=\left(\int_{-\infty}^{\infty} \int_{-\infty}^{\infty}\left|\left(\check{\Phi}_{2 a(t+\theta)}^{L} \otimes \check{\Phi}_{2^{b(s+\gamma)}}^{G}\right) * f(x, y)\right|^{2} d t d s\right)^{1 / 2} .
$$

Then by a well-known argument (see [16, 19]), we obtain

$$
\left\|\mathbf{J}_{\theta, \gamma, \Phi, L, G}^{(a, b)}(f)\right\|_{p} \leq C_{p}\|f\|_{p}
$$

for all $1<p<\infty$.

Now for $p>2$, by an argument similar to that used in the proof of a lemma in [14, page 544], choose a nonnegative function $\omega \in L^{(p / 2)^{\prime}}\left(\mathbb{R}^{N} \times \mathbb{R}^{M}\right)$ such that

$$
\left\|Z_{\sigma, \theta, \gamma, \Phi, L, G}^{(a, b)} f\right\|_{p}^{2}=\int_{\mathbb{R}^{N}} \int_{\mathbb{R}^{M}} \int_{-\infty}^{\infty} \int_{-\infty}^{\infty}\left|\left(\check{\Phi}_{2^{a(t+\theta)}}^{L} \otimes \check{\Phi}_{2^{b(s+\gamma)}}^{G}\right) * \sigma_{t, s} * f(x, y)\right|^{2} \omega(x, y) d t d s .
$$

Thus by condition (a), condition (f), and (2.13), we get

$$
\left\|Z_{\sigma, \theta, \gamma, \Phi, L, G}^{(a, b)} f\right\|_{p} \leq C\left\|\mathbf{J}_{\theta, \gamma, \Phi, L, G}^{(a, b)}(f)\right\|_{p} \sqrt{\left\|M_{\sigma}\right\|_{(p / 2)^{\prime}}}\|f\|_{p} \leq C\|f\|_{p},
$$

where $C$ is a constant independent of the essential variables. On the other hand, by duality we get (2.15) for $1<p<2$. Hence, for all $1<p<\infty$, we have

$$
\left\|Z_{\sigma, \theta, \gamma, \Phi, L, G}^{(a, b)} f\right\|_{p} \leq C\|f\|_{p} .
$$

Next, we claim

$$
\left\|Z_{\sigma, \theta, \gamma, \Phi, L, G}^{(a, b)} f\right\|_{L^{2}\left(\mathbb{R}^{n} \times \mathbb{R}^{m}\right)} \leq C(|\theta||\gamma|)^{-1-\alpha}\|f\|_{L^{2}\left(\mathbb{R}^{n} \times \mathbb{R}^{m}\right)} .
$$

To see (2.17), we only need to apply Plancherel's theorem along with condition (d). Namely, for $t, s, \theta, \gamma \in \mathbb{R}$, let $\mathbf{A}(t, \theta)=\left\{\xi \in \mathbb{R}^{N}: 2^{-a(t+\theta)-1} \leq|L(\xi)| \leq 2^{-a(t+\theta)+1}\right\}$ and let $\mathbf{B}(s, \gamma)=\left\{\eta \in \mathbb{R}^{M}: 2^{-b(s+\gamma)-1} \leq|G(\eta)| \leq 2^{-b(s+\gamma)+1}\right\}$. Then

$$
\begin{aligned}
& \left\|Z_{\sigma, \theta, \gamma, \Phi, L, G}^{(a, b)} f\right\|_{L^{2}\left(\mathbb{R}^{n} \times \mathbb{R}^{m}\right)}^{2} \\
& \leq C \int_{-\infty}^{\infty} \int_{-\infty}^{\infty} \int_{\mathbf{A}(t, \theta)} \int_{\mathbf{B}(s, \gamma)}|\hat{f}(\xi, \eta)|^{2} \\
& \times\left\{\left(\log ^{+}\left|2^{a t} L(\xi)\right|\right)\left(\log ^{+}\left|2^{b s} G(\eta)\right|\right)\right\}^{-2-2 \alpha} d \xi d \eta d t d s \\
& \leq C(|\theta||\gamma|)^{-2-2 \alpha} \int_{-\infty}^{\infty} \int_{-\infty}^{\infty} \int_{\mathbf{A}(t, \theta)} \int_{\mathbf{B}(s, \gamma)}|\hat{f}(\xi, \eta)|^{2} d \xi d \eta d t d s \\
& =C(|\theta||\gamma|)^{-2-2 \alpha}\|f\|_{L^{2}\left(\mathbb{R}^{n} \times \mathbb{R}^{m}\right)}^{2} \text {. }
\end{aligned}
$$

By interpolation between (2.17) and (2.16) for any $p \in((2+2 \alpha) /(1+2 \alpha), 2+2 \alpha)$, there exists $\delta_{p}>1$ such that

$$
\left\|Z_{\sigma, \theta, \gamma, \Phi, L, G}^{(a, b)} f\right\|_{L^{p}\left(\mathbb{R}^{n} \times \mathbb{R}^{m}\right)} \leq C|\theta|^{-\delta_{p}}|\gamma|^{-\delta_{p}}\|f\|_{L^{p}}
$$


Therefore, the boundedness of the operators (2.2)-(2.9) follows by (2.19) and Minkowski's inequality. The boundedness of the operator (2.10) follows by Minkowski's inequality and (2.16). This completes the proof.

Lemma 2.1 immediately implies the following.

LemmA 2.2. Suppose that $N, M, \tilde{N}, \widetilde{M}, a, b, \alpha, \sigma=\left\{\sigma_{t, s}: t, s \in \mathbb{R}\right\}, L$, and $G$ are as in Lemma 2.1. If $\sigma=\left\{\sigma_{t, s}: t, s \in \mathbb{R}\right\}$ satisfies the conditions (a)-(f) of Lemma 2.1, then

$$
\mu_{\sigma}^{(a, b)} f(x, y)=\left(\int_{-\infty}^{\infty} \int_{-\infty}^{\infty}\left|\sigma_{t, s} * f(x, y)\right|^{2} d t d s\right)^{1 / 2}
$$

is bounded on $L^{p}\left(\mathbb{R}^{N} \times \mathbb{R}^{M}\right)$ for all $p \in((2+2 \alpha) /(1+2 \alpha), 2+2 \alpha)$. Moreover the $L^{p}$ bounds are independent of the linear transformations $L$ and $G$.

Proof. Choose a nonnegative $C^{\infty}$ radial function $\Phi$ on $\mathbb{R}$ with $\operatorname{supp}(\Phi) \subset\left[2^{-1}, 2\right]$ and $0 \leq \Phi(t) \leq 1$, and

$$
\int_{0}^{\infty} t^{-1} \Phi(t) d t=1
$$

Then, it is easy to see that

$$
f(x, y)=C \int_{-\infty}^{\infty} \int_{-\infty}^{\infty}\left(\check{\Phi}_{2}^{L}{ }^{a t} \otimes \check{\Phi}_{2^{b s}}^{G}\right) * f(x, y) d t d s,
$$

where $C$ is a constant independent of $L$ and $G$. Therefore, by (2.22), it follows that

$$
\begin{aligned}
\mu_{\sigma}^{(a, b)} f(x, y) \leq C & \left\{\mu_{\sigma, \Phi, L, G}^{(a, b, \infty,++)} f(x, y)+\mu_{\sigma, \Phi, L, G}^{(a, b, \infty,+-)} f(x, y)+\mu_{\sigma, \Phi, L, G}^{(a, b, \infty,--)} f(x, y)\right. \\
& +\mu_{\sigma, \Phi, L, G}^{(a, b, \infty,-+)} f(x, y)+\mu_{\sigma, \Phi, L, G}^{(a, b, 0 \infty,+)} f(x, y)+\mu_{\sigma, \Phi, L, G}^{(a, b, 0 \infty,-)} f(x, y) \\
& \left.+\mu_{\sigma, \Phi, L, G}^{(a, b, \infty 0,-)} f(x, y)+\mu_{\sigma, \Phi, L, G}^{(a, b, \infty 0,+)} f(x, y)+\mu_{\sigma, \Phi, L, G}^{(a, b, 00)} f(x, y)\right\} .
\end{aligned}
$$

Hence the proof is complete by Lemma 2.1.

Now, we have the following lemma which can be proved by a proper modification of the arguments in $[3,15]$.

LEMMA 2.3. Let $M, N, \tilde{N}, \widetilde{M} \geq 1$, and $\left\{\lambda_{t, s}^{(l, r)}: t, s \in \mathbb{R}, 0 \leq l \leq \tilde{N}, 0 \leq r \leq \widetilde{M}\right\}$ be a family of Borel measures on $\mathbb{R}^{N} \times \mathbb{R}^{M}$ with $\lambda_{t, s}^{(0, r)}=0$ and $\sigma_{k, j}^{(l, 0)}=0$ for every $t, s \in \mathbb{R}$. Let $\left\{N_{l}, M_{r}: 1 \leq l \leq \tilde{N}, 1 \leq r \leq \widetilde{M}\right\} \subseteq \mathbb{N}$ and let $L_{l}: \mathbb{R}^{N} \rightarrow \mathbb{R}^{N_{l}}$ and $G_{r}: \mathbb{R}^{M} \rightarrow \mathbb{R}^{M_{r}}$ be linear transformations, $1 \leq l \leq \tilde{N}, 1 \leq r \leq \widetilde{M}$. Suppose that

(i) $\left\|\lambda_{t, s}^{(l, r)}\right\| \leq C$;

(ii) $\left|\hat{\lambda}_{t, s}^{(l, r)}(\xi, \eta)\right| \leq C\left(\log ^{+}\left|2^{l t} L_{l}(\xi)\right|\right)^{-1-\alpha}\left(\log ^{+}\left|2^{r s} G_{r}(\eta)\right|\right)^{-1-\alpha}$;

(iii) $\left|\hat{\lambda}_{t, s}^{(l, r)}(\xi, \eta)-\hat{\lambda}_{t, s}^{(l-1, r)}(\xi, \eta)\right| \leq C\left|2^{l t} L_{l}(\xi)\right|\left(\log ^{+}\left|2^{r s} G_{r}(\eta)\right|\right)^{-1-\alpha}$;

(iv) $\left|\hat{\lambda}_{t, s}^{(l, r)}(\xi, \eta)-\hat{\lambda}_{t, s}^{(l, r-1)}(\xi, \eta)\right| \leq C\left(\log ^{+}\left|2^{l t} L_{l}(\xi)\right|\right)^{-1-\alpha} \mid 2^{r s} G_{r}(\eta) \|$;

(v) $\left|\hat{\lambda}_{t, s}^{(l, r)}(\xi, \eta)-\hat{\lambda}_{t, s}^{(l-1, r)}(\xi, \eta)-\hat{\lambda}_{t, s}^{(l, r-1)}(\xi, \eta)+\hat{\lambda}_{t, s}^{(l-1, r-1)}(\xi, \eta)\right| \leq C\left|2^{l t} L_{l}(\xi)\right|\left|2^{r s} G_{r}(\eta)\right|$;

(vi) $\left|\hat{\lambda}_{t, s}^{(l, r-1)}(\xi, \eta)-\hat{\lambda}_{t, s}^{(l-1, r-1)}(\xi, \eta)\right| \leq C\left|2^{l t} L_{l}(\xi)\right|$;

(vii) $\left|\hat{\lambda}_{t, s}^{(l-1, r)}(\xi, \eta)-\hat{\lambda}_{t, s}^{(l-1, r-1)}(\xi, \eta)\right| \leq C\left|2^{r s} G_{r}(\eta)\right|$; 
(viii) $\left\|M_{\lambda^{(l, r)}} f\right\|_{p} \leq C_{p}\|f\|_{p}$ for all $1<p<\infty$, where $M_{\lambda^{(l, r)}}$ is given by (2.11) with $\sigma_{t, s}$ replaced by $\lambda_{t, s}^{(l, r)}$.

Then there exists a family of Borel measures $\left\{\sigma_{t, s}^{(l, r)}: t, s \in \mathbb{R}, 1 \leq l \leq \tilde{N}, 1 \leq r \leq \widetilde{M}\right\}$ on $\mathbb{R}^{N} \times \mathbb{R}^{M}$ such that

$$
\lambda_{t, s}^{(\widetilde{N}, \widetilde{M})}=\sum_{l=1}^{\widetilde{N}} \sum_{r=1}^{\widetilde{M}} \sigma_{t, s}^{(l, r)} \quad \forall t, s \in \mathbb{R},
$$

and that for each $1 \leq l \leq \tilde{N}, 1 \leq r \leq \widetilde{M}$, the family $\left\{\sigma_{t, s}^{(l, r)}: t, s \in \mathbb{R}\right\}$ satisfies the assumptions (a)-(f) of Lemma 2.1.

\section{Proof of Theorem 1.2.}

Proof. Let $n, m \geq 2, N, M, \tilde{N}, \widetilde{M} \geq 1$. Let $\Omega \in \bigcap_{l=1}^{\widetilde{N}} \cap_{r=1}^{\widetilde{M}} F_{\alpha}\left(\mathbf{S}^{n-1}, \mathbf{S}^{m-1}, l, r\right)$ for some $\alpha>0$. Let $\mathscr{P}=\left(P_{1}, \ldots, P_{N}\right) \in(\mathscr{V}(n, \tilde{N}))^{N}$ and $\mathscr{2}=\left(Q_{1}, \ldots, Q_{M}\right) \in(\mathscr{V}(m, \widetilde{M}))^{M}$. Then the polynomials $P_{1}, \ldots, P_{N}, Q_{1}, \ldots, Q_{M}$ can be written as

$$
\begin{aligned}
P_{j}(y) & =\sum_{|\beta| \leq \tilde{N}} a_{\beta, j} y^{\beta}, \quad j=1, \ldots, N, \\
Q_{k}(y) & =\sum_{|\widetilde{\beta}| \leq \widetilde{M}} b_{\widetilde{\beta}, k} y^{\widetilde{\beta}}, \quad k=1, \ldots, M,
\end{aligned}
$$

where $\beta \in(\mathbb{N} \cup\{0\})^{N}$ and $\widetilde{\beta} \in(\mathbb{N} \cup\{0\})^{M}$.

For each $1 \leq l \leq \tilde{N}$ and $1 \leq r \leq \widetilde{M}$, let

$$
\begin{gathered}
A(l)=\left\{\beta \in(\mathbb{N} \cup\{0\})^{N}:|\beta|=l\right\}, \\
B(r)=\left\{\tilde{\beta} \in(\mathbb{N} \cup\{0\})^{M}:|\tilde{\beta}|=r\right\} .
\end{gathered}
$$

Let $N_{l}=|A(l)|$ and $M_{r}=|B(r)|$. For each $\beta \in A(l)$ and $\widetilde{\beta} \in B(r)$, let

$$
\overrightarrow{a_{\beta, l}}=\left(a_{\beta, 1}, \ldots, a_{\beta, N}\right), \quad \overrightarrow{b_{\widetilde{\beta}, r}}=\left(b_{\widetilde{\beta}, 1}, \ldots, a_{\widetilde{\beta}, M}\right) .
$$

Define the linear transformations $L_{l}=\left(L_{\beta l}\right)_{\beta \in A(l)}: \mathbb{R}^{N} \rightarrow \mathbb{R}^{N_{l}}$ and $G_{r}=\left(G_{\tilde{\beta} r}\right)_{\tilde{\beta} \in B(r)}$ : $\mathbb{R}^{M} \rightarrow \mathbb{R}^{M_{r}}$ by

$$
\begin{aligned}
L_{\beta l}(\xi) & =\xi \cdot \overrightarrow{a_{\beta, l}}, \\
G_{\tilde{\beta} r}(\eta) & =\eta \cdot \overrightarrow{b_{\tilde{\beta}, r}} .
\end{aligned}
$$

Without loss of generality, we will assume that $L_{l}$ and $G_{r}$ are nonzero for all $1 \leq l \leq \tilde{N}$ and $1 \leq r \leq \widetilde{M}$. 
For $1 \leq l \leq \tilde{N}$ and $1 \leq r \leq \widetilde{M}$, let $\mathscr{P}_{l}(u)=\left(P_{1, l}(u), \ldots, P_{N, l}(u)\right)$ and $\mathscr{2}_{r}(v)=\left(Q_{1, r}(v), \ldots\right.$, $\left.Q_{M, r}(v)\right)$, where

$$
\begin{aligned}
P_{j, l}(u) & =\sum_{|\beta| \leq l} a_{\beta, j} u^{\beta}, \quad j=1, \ldots, N, \\
Q_{k, r}(v) & =\sum_{|\widetilde{\beta}| \leq r} b_{\widetilde{\beta}, k} v^{\widetilde{\beta}}, \quad k=1, \ldots, M .
\end{aligned}
$$

Also, let

$$
\begin{aligned}
& \mathscr{H}_{\mathscr{P}_{l}}\left(u^{\prime}\right)=N_{l}^{-1 / 2}\left(\left(u^{\prime}\right)^{\beta}\right)_{\beta \in A(l)}, \\
& \mathscr{H}_{\mathscr{Q}_{r}}\left(v^{\prime}\right)=M_{r}^{-1 / 2}\left(\left(v^{\prime}\right)^{\tilde{\beta}}\right)_{\tilde{\beta} \in B(r)} .
\end{aligned}
$$

Then clearly

$$
\mathscr{H}^{\mathscr{P}_{l}} \in\left(\mathscr{H}^{I}(n, l)\right)^{N_{l}}, \quad \mathscr{H}_{\mathscr{Q}_{r}} \in\left(\mathscr{H}^{I}(m, r)\right)^{M_{r}} .
$$

Let $\sigma=\left\{\lambda_{t, s}^{(l, r)}: t, s \in \mathbb{R}\right\}$ be the family measures where $\sigma_{t, s}$ is given by

$$
\iint f d \lambda_{t, s}^{(l, r)}=2^{-t-s} \int_{|u| \leq 2^{t}} \int_{|v| \leq 2^{s}} f\left(\mathscr{P}_{l}(u), \mathscr{2}_{r}(v)\right) \frac{\Omega(u, v) d u d v}{|u|^{n-1}|v|^{m-1}} .
$$

Then it is clear that

$$
M_{\Omega, \mathscr{P}, 2} f(x, y)=\left(\int_{-\infty}^{\infty} \int_{-\infty}^{\infty}\left|\left(\lambda_{t, s}^{(\tilde{N}, \widetilde{M})} * f\right)(x, y)\right|^{2} d t d s\right)^{1 / 2} .
$$

Therefore, by Lemmas 2.1, 2.2, and 2.3, it suffices to verify that the measures $\left\{\lambda_{t, s}^{(l, r)}\right.$ : $t, s \in \mathbb{R}, 0 \leq l \leq \tilde{N}, 0 \leq r \leq \widetilde{M}\}$ satisfy the assumptions of Lemma 2.3 .

For $t, s \in \mathbb{R}, 0 \leq l \leq \tilde{N}, 0 \leq r \leq \widetilde{M}$, let $I_{l}(t, \xi, x)$ and $J_{r}(s, \eta, y)$ be the complex-valued functions defined on $\mathbb{R} \times \mathbb{R}^{N} \times \mathbf{S}^{n-1}$ and $\mathbb{R} \times \mathbb{R}^{M} \times \mathbf{S}^{m-1}$, respectively, by

$$
I_{l}\left(t, \xi, u^{\prime}\right)=\int_{0}^{1} e^{-i \xi_{\Phi_{l}}\left(2^{t} u^{\prime} w\right)} d w, \quad J_{r}\left(s, \eta, v^{\prime}\right)=\int_{0}^{1} e^{-i \eta \cdot 2_{r}\left(2^{s} v^{\prime} w\right)} d w .
$$

First, notice that $\left(d^{l} / d w^{l}\right)\left(\xi \mathscr{P}_{l}\left(2^{t} u^{\prime} w\right)\right)=l ! 2^{l t} N_{l}^{1 / 2} L_{l}(\xi) \cdot \mathscr{H}^{\prime} \mathscr{P}_{l}\left(u^{\prime}\right)$ and $\left(d^{r} / d w^{r}\right)(\eta$. $\left.2_{r}\left(2^{s} v^{\prime} w\right)\right)=r ! 2^{r s} M_{r}^{1 / 2} G_{r}(\eta) \cdot \mathscr{H}_{2}\left(v^{\prime}\right)$. Thus, by the van der Corput lemma [19], we obtain

$$
\begin{aligned}
& \left|I_{l}\left(t, \xi, u^{\prime}\right)\right| \leq C\left|2^{l t} N_{l}^{1 / 2} L_{l}(\xi) \cdot \mathscr{H}_{\mathscr{P}_{l}}\left(u^{\prime}\right)\right|^{-1 / l}, \\
& \left|J_{r}\left(s, \eta, v^{\prime}\right)\right| \leq C\left|2^{r s} M_{r}^{1 / 2} G_{r}(\eta) \cdot \mathscr{H}_{2}\left(v^{\prime}\right)\right|^{-1 / r},
\end{aligned}
$$

which when interpolated with the trivial estimates $\left|I_{l}\left(t, \xi, u^{\prime}\right)\right| \leq C$ and $\left|J_{r}\left(s, \eta, v^{\prime}\right)\right| \leq C$, respectively, imply that

$$
\begin{aligned}
& \left|I_{l}\left(t, \xi, u^{\prime}\right)\right| \leq C\left|2^{l t} N_{l}^{1 / 2} L_{l}(\xi) \cdot \mathscr{H}_{\mathscr{P}_{l}}\left(u^{\prime}\right)\right|^{-1 / 2 l}, \\
& \left|J_{r}\left(s, \eta, v^{\prime}\right)\right| \leq C\left|2^{r s} M_{r}^{1 / 2} G_{r}(\eta) \cdot \mathscr{H}_{2_{r}}\left(v^{\prime}\right)\right|^{-1 / 2 r} .
\end{aligned}
$$


On the other hand, it is straightforward to see that the following hold:

$$
\begin{aligned}
\left|I_{l}(t, \xi, x)-I_{l-1}\left(t, \xi, u^{\prime}\right)\right| & \leq\left|2^{l t} N_{l}^{1 / 2} L_{l}(\xi)\right|, \\
\left|J_{r}\left(s, \eta, v^{\prime}\right)-J_{r-1}\left(s, \eta, v^{\prime}\right)\right| & \leq\left|2^{r s} M_{r}^{1 / 2} G_{r}(\eta)\right|, \\
\left|I_{l}\left(t, \xi, u^{\prime}\right)\right| & \leq 1, \\
\left|J_{r}\left(s, \eta, v^{\prime}\right)\right| & \leq 1
\end{aligned}
$$

for $1 \leq l \leq \tilde{N}, 1 \leq r \leq \widetilde{M}$.

Now, clearly condition (i) holds trivially. Next, by combining (3.12), (3.15), and (3.16), we obtain

$$
\begin{aligned}
& \left|I_{l}\left(t, \xi, u^{\prime}\right)\right| \leq C\left(\log ^{+}\left|2^{l t} L_{l}(\xi)\right|\right)^{-1-\alpha}\left(l+\alpha+\log ^{+}\left|\frac{1}{\left(L_{l}(\xi)\right)^{\prime} \cdot \mathscr{H}_{\mathscr{P}_{l}\left(u^{\prime}\right)}}\right|\right)^{1+\alpha}, \\
& \left|J_{r}\left(s, \eta, v^{\prime}\right)\right| \leq C\left(\log ^{+}\left|2^{r s} G_{r}(\eta)\right|\right)^{-1-\alpha}\left(r+\alpha+\log ^{+}\left|\frac{1}{\left(G_{r}(\eta)\right)^{\prime} \cdot \mathscr{H}_{2_{r}}\left(v^{\prime}\right)}\right|\right)^{1+\alpha} .
\end{aligned}
$$

Therefore, since $\left(L_{l}(\xi)\right)^{\prime} \cdot \mathscr{H}_{\mathscr{P}_{l}}(\cdot) \in \mathscr{H}^{I}(n, l),\left(G_{r}(\eta)\right)^{\prime} \cdot \mathscr{H}_{\mathscr{Q}_{r}}\left(v^{\prime}\right) \in \mathscr{H}^{I}(m, r)$, and $\Omega \in$ $F_{\alpha}\left(\mathbf{S}^{n-1}, \mathbf{S}^{m-1}, l, r\right)$ for all $1 \leq l \leq \tilde{N}, 1 \leq r \leq \widetilde{M}$, we immediately obtain the estimates (ii), (iii), (iv), (v), (vi), and (vii) by a proper use of the estimates (3.13)-(3.18). We omit the details.

Finally, we turn to the proof of (viii). It can be easily verified that

$$
M_{\lambda(l, r)}(f)(x, y) \leq \int_{\mathbf{S}^{n-1}} \int_{\mathbf{S}^{m-1}}|\Omega(u, v)|\left(M_{\Phi_{l}, u^{\prime}}^{1} \circ M_{2_{r}, v^{\prime}}^{2}\right)(f)(x, y) d \sigma(u) d \sigma(v),
$$

where

$$
\begin{aligned}
& M_{\mathscr{P}_{l}, u^{\prime}}^{1} f(x, y)=\sup _{h>0} h^{-1} \int_{|t|<h}\left|f\left(x-\mathscr{P}_{l}\left(t u^{\prime}\right), y\right)\right| d t, \\
& M_{\mathscr{Q}_{r}, v^{\prime}}^{2} f(x, y)=\sup _{h>0} h^{-1} \int_{|s|<h}\left|f\left(x, y-\mathscr{2}_{r}\left(s v^{\prime}\right)\right)\right| d s,
\end{aligned}
$$

and $\circ$ denotes the composition of operators. Thus, the estimate (viii) follows by (3.19), Hölder's inequality, and [19, Proposition 1, page 477]. This completes the verification of the assumptions of Lemma 2.3 and hence the proof.

4. Proof of Corollary 1.3. Corollary 1.3 is a simple consequence of Theorem 1.2 and the following lemma.

LEMMA 4.1. $\bigcap_{l=1}^{\infty} \bigcap_{r=1}^{\infty} F_{\alpha}\left(\mathbf{S}^{1}, \mathbf{S}^{1}, l, r\right)=F_{\alpha}\left(\mathbf{S}^{1}, \mathbf{S}^{1}, 1,1\right)$.

A proof of Lemma 4.1 can be obtained by adapting the one parameter argument in [6]. For readers convenience, details are presented below.

We will need the following lemma (see [6, Lemma 3.1]). 
LEMMA 4.2 [6]. Let $m \in N, a_{0}, a_{1}, \ldots, a_{m} \in \mathcal{C}$ and $g(z)=a_{0}+a_{1} z+\cdots+a_{m} z^{m}$ for $z \in C$. If $z_{1}, \ldots, z_{l}$ are the roots of $g(z)$ which lie in $\{z \in C:|z| \leq 2\}$, then

$$
|g(z)| \geq 6^{-m}\left(\sup _{|z|=1}|g(z)|\right) \prod_{s=1}^{l}\left|z-z_{s}\right|
$$

holds for $|z| \leq 1$.

Proof OF LEMma 4.1. Since $\bigcap_{l=1}^{\infty} \bigcap_{r=1}^{\infty} F_{\alpha}\left(\mathbf{S}^{1}, \mathbf{S}^{1}, l, r\right) \subset F_{\alpha}\left(\mathbf{S}^{1}, \mathbf{S}^{1}, 1,1\right)$, it suffices to show that $F_{\alpha}\left(\mathbf{S}^{1}, \mathbf{S}^{1}, 1,1\right) \subset \bigcap_{l=1}^{\infty} \bigcap_{r=1}^{\infty} F_{\alpha}\left(\mathbf{S}^{1}, \mathbf{S}^{1}, l, r\right)$ for all $\alpha>0$. Let $\Omega \in F_{\alpha}\left(\mathbf{S}^{1}, \mathbf{S}^{1}, 1,1\right)$. First, as in the one parameter case in [17], we observe that the condition $\Omega \in F_{\alpha}\left(\mathbf{S}^{1}\right.$, $\left.\mathbf{S}^{1}, 1,1\right)$ is equivalent to the following condition:

$$
\sup _{\theta, \phi \in[0,2 \pi]} \iint_{[0,2 \pi]^{2}}|\Omega(t, s)|\{G(t, s, \theta, \phi)\}^{1+\alpha} d t d s<\infty,
$$

where

$$
G(t, s, \theta, \phi)=\log ^{+}\left(\frac{1}{|t-\theta||s-\phi|}\right)+\log ^{+}\left(\frac{1}{|t-\theta|}\right) \log ^{+}\left(\frac{1}{|s-\phi|}\right) .
$$

Let $l, r \in \mathbb{N}$. Then there exist $\lambda_{l}>0$ and $\mu_{r}>0$ such that

$$
\begin{aligned}
& \sup _{u \in \mathbf{S}^{1}}|P(u)| \geq \lambda_{l}\|P\|, \\
& \sup _{v \in \mathbf{S}^{1}}|Q(v)| \geq \mu_{r}\|Q\|
\end{aligned}
$$

hold for all $P \in \mathscr{V}(2, l)$ and $Q \in \mathscr{V}(2, r)$.

For $P \in \mathscr{V}(2, l)$ and $Q \in \mathscr{V}(2, r)$ with $\|P\|=\|Q\|=1$, we write

$$
\begin{aligned}
& P(u)=P\left(u_{1}, u_{2}\right)=\sum_{j+k=l} a_{j k} u_{1}^{j} u_{2}^{k}, \\
& Q(v)=Q\left(v_{1}, v_{2}\right)=\sum_{j+k=r} b_{j k} v_{1}^{j} v_{2}^{k} .
\end{aligned}
$$

Then, by (4.4), and similar argument as in [6], there exist $k_{1}, k_{2} \in \mathbb{N},\left\{z_{1}, \ldots, z_{k_{1}}\right\} \subset \mathbf{S}^{1}$, $\left\{w_{1}, \ldots, w_{k_{2}}\right\} \subset \mathbf{S}^{1}$, and constants $C_{l}=C(l)$ and $C_{r}=C(r)$ such that

$$
\begin{aligned}
& |P(u)| \geq C_{l} \prod_{s=1}^{k_{1}}\left|u-z_{s}\right|, \\
& |Q(v)| \geq C_{r} \prod_{s=1}^{k_{2}}\left|v-w_{s}\right| .
\end{aligned}
$$

Hence, by (4.2), (4.6), we get $\Omega \in F_{\alpha}\left(\mathbf{S}^{1}, \mathbf{S}^{1}, l, r\right)$. This completes the proof. 
ACKNOWLEDGMENT. The author would like to thank the referees of this note for their valuable remarks and comments.

\section{REFERENCES}

[1] H. Al-Qassem and A. Al-Salman, Rough Marcinkiewicz integral operators, Int. J. Math. Math. Sci. 27 (2001), no. 8, 495-503.

[2] H. Al-Qassem, A. Al-Salman, L. Cheng, and Y. Pan, Marcinkiewicz integrals on product domains, to appear in Studia Math.

[3] H. Al-Qassem and Y. Pan, $L^{p}$ boundedness for singular integrals with rough kernels on product domains, Hokkaido Math. J. 31 (2002), no. 3, 555-613.

[4] A. Al-Salman and H. Al-Qassem, Integral operators of Marcinkiewicz type, J. Integral Equations Appl. 14 (2002), no. 4, 343-354.

[5] A. Al-Salman, H. Al-Qassem, L. Cheng, and Y. Pan, $L^{p}$ bounds for the function of Marcinkiewicz, Math. Res. Lett. 9 (2002), no. 5-6, 697-700.

[6] A. Al-Salman and Y. Pan, Singular integrals with rough kernels, Canad. Math. Bull. 47 (2004), no. 1, 3-11.

[7] A. Benedek, A. Calderón, and R. Panzone, Convolution operators on Banach space valued functions, Proc. Nat. Acad. Sci. USA 48 (1962), 356-365.

[8] J. Chen, D. Fan, and Y. Pan, A note on a Marcinkiewicz integral operator, Math. Nachr. 227 (2001), 33-42.

[9] J. Chen, D. Fan, and Y. Ying, Rough Marcinkiewicz integrals with $L\left(\log ^{+} L\right)^{2}$ kernels on product spaces, Adv. Math. (China) 30 (2001), no. 2, 179-181.

[10] _ The method of rotation and Marcinkiewicz integrals on product domains, Studia Math. 153 (2002), no. 1, 41-58.

[11] Y. Choi, Marcinkiewicz integrals with rough homogeneous kernels of degree zero in product domains, J. Math. Anal. Appl. 261 (2001), no. 1, 53-60.

[12] Y. Ding, $L^{2}$-boundedness of Marcinkiewicz integral with rough kernel, Hokkaido Math. J. 27 (1998), no. 1, 105-115.

[13] Y. Ding, D. Fan, and Y. Pan, On the $L^{p}$ boundedness of Marcinkiewicz integrals, Michigan Math. J. 50 (2002), no. 1, 17-26.

[14] J. Duoandikoetxea and J. L. Rubio de Francia, Maximal and singular integral operators via Fourier transform estimates, Invent. Math. 84 (1986), no. 3, 541-561.

[15] D. Fan and Y. Pan, Singular integral operators with rough kernels supported by subvarieties, Amer. J. Math. 119 (1997), no. 4, 799-839.

[16] R. Fefferman and E. M. Stein, Singular integrals on product spaces, Adv. in Math. 45 (1982), no. 2, 117-143.

[17] L. Grafakos and A. Stefanov, $L^{p}$ bounds for singular integrals and maximal singular integrals with rough kernels, Indiana Univ. Math. J. 47 (1998), no. 2, 455-469.

[18] E. M. Stein, On the functions of Littlewood-Paley, Lusin, and Marcinkiewicz, Trans. Amer. Math. Soc. 88 (1958), 430-466.

[19] _ Harmonic Analysis: Real-Variable Methods, Orthogonality, and Oscillatory Integrals, Princeton Mathematical Series, vol. 43, Princeton University Press, New Jersey, 1993.

Ahmad Al-Salman: Department of Mathematics, Yarmouk University, Irbid, Jordan E-mail address: a1sa7man@yu. edu. jo 


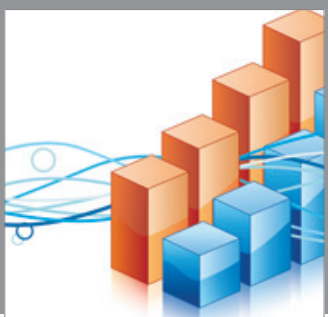

Advances in

Operations Research

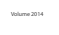

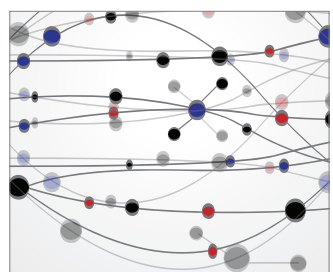

\section{The Scientific} World Journal
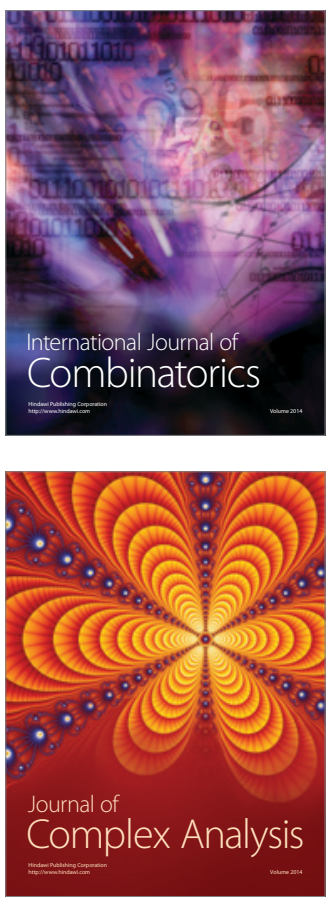

International Journal of

Mathematics and

Mathematical

Sciences
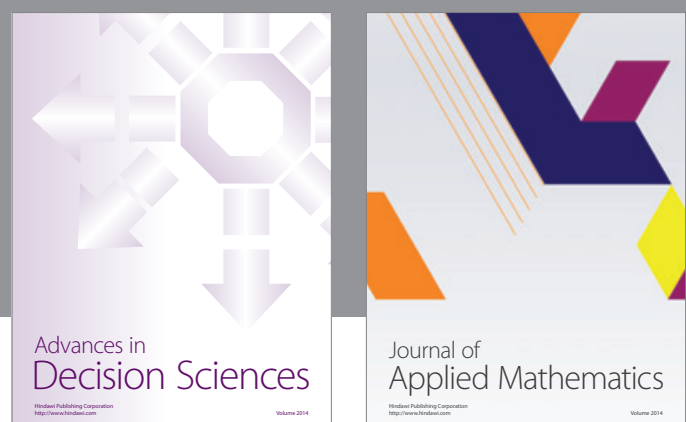

Journal of

Applied Mathematics
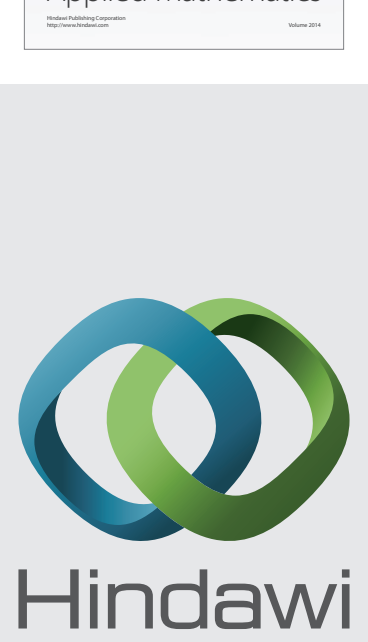

Submit your manuscripts at http://www.hindawi.com
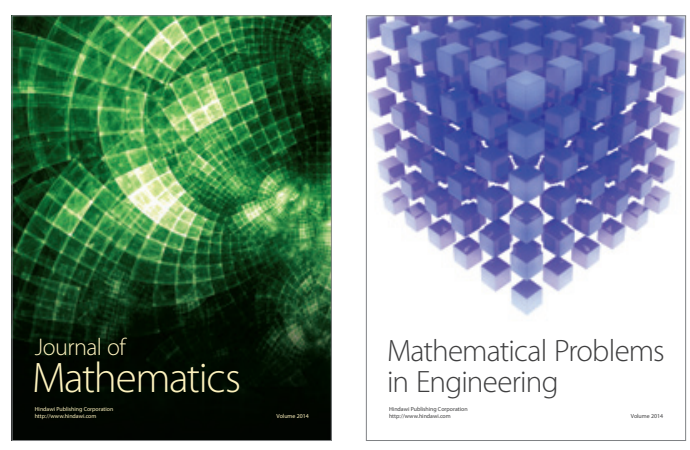

Mathematical Problems in Engineering
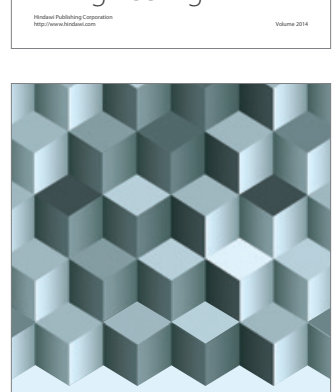

Journal of

Function Spaces
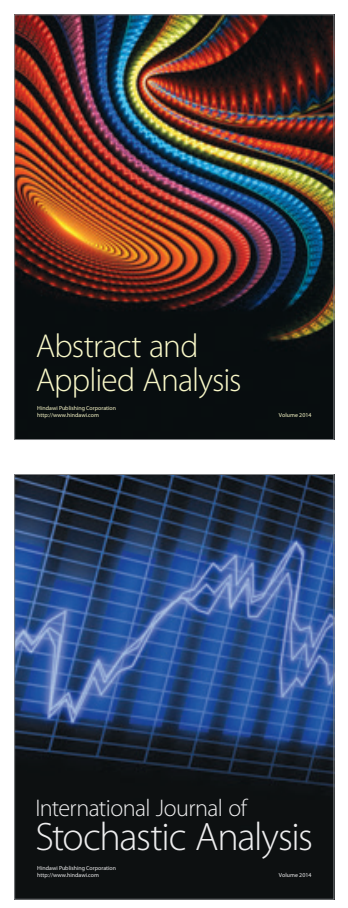

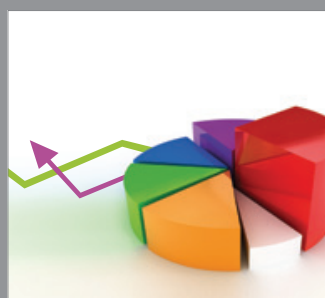

ournal of

Probability and Statistics

Promensencen
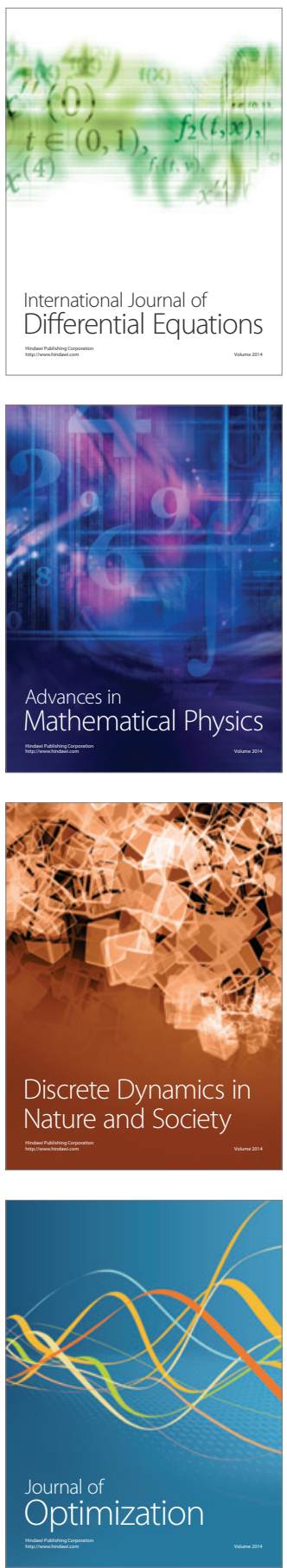